\title{
Bounds on the Distribution of a Sum of Correlated Lognormal Random Variables and Their Application
}

\author{
C. Tellambura, Senior Member, IEEE
}

\begin{abstract}
The cumulative distribution function (cdf) of a sum of correlated or even independent lognormal random variables (RVs), which is of wide interest in wireless communications, remains unsolved despite long standing efforts. Several cdf approximations are thus widely used. This letter derives bounds for the cdf of a sum of 2 or 3 arbitrarily correlated lognormal RVs and of a sum of any number of equally-correlated lognormal RVs. The bounds are single-fold integrals of readily computable functions and extend previously known bounds for independent lognormal summands. An improved set of bounds are also derived which are expressed as 2 -fold integrals. For correlated lognormal fading channels, new expressions are derived for the moments of the output SNR and amount of fading for maximal ratio combining (MRC), selection combining (SC) and equal gain combining (EGC) and outage probability expressions for SC.
\end{abstract}

Index Terms-Amount of fading, cochannel interference, lognormal distribution, diversity combining.

\section{INTRODUCTION}

A

LOGNORMAL power sum of type

$$
I=\sum_{i=1}^{n} e^{X_{i}}
$$

where each $X_{i}$ is a Gaussian random variable (RV) appears pervasively in wireless communications. Applications include modeling and assessing cochannel interference, evaluating coverage for cellular mobile networks and modeling fading and shadowing [1], [2], and the book by Aitchison and Brown [3] lists over 100 applications. Nevertheless, no exact closedform formula for the distribution of $I$ is known. Even the characteristic function (chf) of a lognormal RV is not known in closed-form. Thus, several approximations have been developed such as moment matching [4], cumulant matching [5] and recursive methods [6] - for comparisons see [2] [7]. For more recent results on lognormal sums the reader is referred to [8]-[17].

Although independent lognormal sums have been widely studied, applications where correlation among the summands in (1) exists occur just as frequently. Therefore, the distribution of correlated lognormal sums has applications such as macro-diversity systems [18], soft handoff algorithms, single frequency networks and others. Moment matching or cumulant matching approximations that have been developed for independent lognormal sums can also be extended for correlated lognormal sums [19]-[21].

Paper approved by F. Santucci, the Editor for Wireless System Performance of the IEEE Communications Society. Manuscript received December 15, 2003; revised September 16, 2006. This work has been supported in part by NSERC and iCORE.

C. Tellambura is with the Department of Electrical and Computer Engineering, University of Alberta, Edmonton, AB, Canada T6G 2V4 (e-mail: chintha@ece.ualberta.ca).

Digital Object Identifier 10.1109/TCOMM.2008.030947.
In this paper, we derive bounds for the cdf of a sum of two or three correlated lognormal RVs with arbitrary correlation and of a sum of any number of equally-correlated lognormal RVs. Several previously derived bounds are shown to be special cases of these. A further set of improved bounds (2-fold integrals) are derived. The simulation results confirm the tightness of these bounds. Although diversity combiners such as maximal ratio combining (MRC), selection combining (SC) and equal gain combining (EGC) have been widely studied over various channel models, their performance over correlated lognormal channels is not available in detail. For correlated lognormal fading channels, we also derive new expressions for the moments of the output SNR and amount of fading for MRC, SC and EGC and outage probability expressions for SC. For this development, we utilize S-function and T-function [22], [23], which are generalizations of the wellknown Q-function and are not known widely in the wireless community. These results facilitate the rapid evaluation of the performance of diversity schemes over correlated lognormal channels, where no results have been published for diversity order more than two.

Our bounds generalize previously published bounds for independent lognormal sums by Slimane [24] and Farley's lower bound (or approximation) for independent lognormal sums [1] [2]. Abu-Dayya \& Beaulieu [19] provide a detailed analysis of outage probabilities in the presence of multiple correlated lognormal interferers, using Wilkinson's approach, Schwartz and Yeh's approach and cumulant matching. Outage probability estimation in the presence of multiple lognormal components has been discussed by Ligeti [21]. Pratesi et al [20] treat outage analysis in mobile radio with generically correlated lognormal interferers. They extend several approximations for the independent case to those for the generically correlated case. Berggren and Slimane [25], by applying the arithmetic-geometric mean inequality, give a lower bound expressed by a single Q-function. Our work is different from all these in that we develop bounds for the correlated case and analyze diversity schemes. A detailed performance analysis of dual-branch diversity schemes over correlated lognormal fading has been developed by Alouini \& Simon [26]. Our results generalize some of their results to multibranch $(n>2)$ diversity systems.

This paper is organized as follows. Section II lists several relevant results for the multinormal distribution. Section III develops bounds for sums of correlated lognormal variables. Section IV derives the performance of SC, MRC and EGC receivers over correlated lognormal channels and Section V concludes the paper. 


\section{NOTATION AND PRELIMINARIES}

To derive bounds for correlated lognormal sums, a number of distributional results on correlated normal RVs are required. This section lists these required results.

\section{A. Multivariate normal distribution}

Let random variables $\mathbf{X}=\left(X_{1}, X_{2}, \cdots, X_{n}\right)^{T}$ have a joint multivariate normal distribution with the joint probability density function (pdf) [27]

$f_{\mathbf{X}}(\mathbf{x})=(2 \pi)^{-n / 2}|\mathbf{R}|^{-1 / 2} \exp \left\{-\frac{1}{2}(\mathbf{x}-\boldsymbol{\mu})^{T} \mathbf{R}^{-1}(\mathbf{x}-\boldsymbol{\mu})\right\}$

where $\mathbf{x}=\left(x_{1}, x_{2}, \cdots, x_{n}\right)^{T}, \boldsymbol{\mu}=\left(\mu_{1}, \mu_{2}, \cdots, \mu_{n}\right)^{T}$, $\mu_{i}=E\left(x_{i}\right), \mathbf{R}=E\left[(\mathbf{x}-\boldsymbol{\mu})(\mathbf{x}-\boldsymbol{\mu})^{T}\right]$ and $|\mathbf{R}|$ denotes the determinant of $\mathbf{R}$. The moment generating function (mgf) of $\mathrm{X}$ is given by

$$
\begin{aligned}
M_{\mathbf{X}}\left(t_{1}, t_{2}, \cdots, t_{n}\right) & =E\left[e^{\left.\mathbf{t}^{T} \cdot \mathbf{X}_{1}\right]}\right. \\
& =\exp \left(\mathbf{t}^{T} \cdot \boldsymbol{\mu}+\frac{1}{2} \mathbf{t}^{T} \mathbf{R} \mathbf{t}\right) .
\end{aligned}
$$

We write $X \sim N\left(\mu, \sigma^{2}\right)$ to denote that $X$ is Gaussian distributed with mean $\mu$ and variance $\sigma^{2}$.

\section{B. Single and bivariate normal distribution functions}

The standardized normal cdf (i.e. that of $X \sim N(0,1)$ ) is denoted by

$$
\Phi(h)=\operatorname{Pr}(X \leq h)=\int_{-\infty}^{h} \phi(t) d t
$$

where $\phi(t)=e^{-t^{2} / 2} / \sqrt{2 \pi}$ is the unit Gaussian pdf. The cdf in (4) relates to the more familiar $Q$ function as $\Phi(h)=1-Q(h)$. Note also that $\Phi(-h)=1-\Phi(h)=Q(h)$. We use the same $\Phi(\cdot)$ to denote the cdf for univariate, bivariate and trivariate cases and the number of arguments of $\Phi(\cdot)$ will indicate which particular case being handled.

The joint cdf of the standardized bivariate normal distribution (i.e. $X_{1}, X_{2} \sim N(0,1)$ and $E\left[X_{1} X_{2}\right]=\rho$ ) is

$$
\begin{gathered}
\Phi\left(h_{1}, h_{2} ; \rho\right)=\frac{1}{2 \pi \sqrt{1-\rho^{2}}} \\
\cdot \int_{-\infty}^{h_{1}} \int_{-\infty}^{h_{2}} \exp \left\{\frac{-\left(x_{1}^{2}-2 \rho x_{1} x_{2}+x^{2}\right)}{2\left(1-\rho^{2}\right)}\right\} d x_{1} d x_{2} .
\end{gathered}
$$

Many numerical methods have been derived to compute the bivariate normal cdf [27]. Using the method due to Owen [23], the bivariate cdf $\Phi\left(h_{1}, h_{2} ; \rho\right)$ can be computed as a sum of simple integrals. An equivalent representation has been derived by Simon [28] and Simon and Alouini [26] used it to analyze dual-diversity SC over correlated lognormal fading. For brevity, we do not list the details here. Note that the bivariate $Q$ function has been studied in [28]. The relationship between $Q\left(h_{1}, h_{2} ; \rho\right)=\operatorname{Pr}\left[X_{1}>h_{1}, X_{2}>h_{2}\right]$ and the bivariate cdf $(5)$ is

$$
\Phi\left(h_{1}, h_{2} ; \rho\right)=1-Q\left(h_{1}\right)-Q\left(h_{2}\right)+Q\left(h_{1}, h_{2} ; \rho\right) .
$$

\section{Trivariate normal distribution function}

We next consider the standardized trivariate cdf, in which $X_{i} \sim N(0,1), i=1,2,3$ and $E\left[X_{i} X_{j}\right]=\rho_{i j}$. The trivariate cdf has been derived by Steck [22]. In the following, we update Steck's notation and note that the cdf is now expressed in form of $\Phi, T$-function and $S$-function. This will yield the cdf as a sum of several single-fold integrals of well-known functions. For $h_{1}, h_{2}, h_{3} \geq 0$ (or $h_{1}, h_{2}, h_{3} \leq 0$ ), the trivariate cdf is given by [22]

$$
\begin{aligned}
& \Phi\left(h_{1}, h_{2}, h_{3} ; \rho_{12}, \rho_{13}, \rho_{23}\right) \\
& =\operatorname{Pr}\left(X_{1}<h_{1}, X_{2}<h_{2}, X_{3}<h_{3}\right) \\
& =\frac{1}{2} \sum_{k=1}^{3}\left(1-\delta_{a_{k} c_{k}}\right) \Phi\left(h_{k}\right) \\
& \quad-\frac{1}{2} \sum_{k=1}^{3}\left[T\left(h_{k}, a_{k}\right)+T\left(h_{k}, c_{k}\right)\right] \\
& \quad-\sum_{k=1}^{3}\left[S\left(h_{k}, a_{k}, b_{k}\right)+S\left(h_{k}, c_{k}, d_{k}\right)\right],
\end{aligned}
$$

where $a_{k}, b_{k}, c_{k}$ and $d_{k}$ are given by Eq. (11),

$$
\begin{aligned}
T(\alpha, \beta) & =\frac{1}{2 \pi} \int_{0}^{\beta} \frac{\exp \left[-\frac{1}{2} \alpha^{2}\left(1+x^{2}\right)\right]}{\left(1+x^{2}\right)} d x \\
& =\frac{1}{2 \pi} \int_{\tan ^{-1}(1 / \beta)}^{\frac{\pi}{2}} \exp \left(-\frac{\alpha^{2}}{2 \sin ^{2} \theta}\right) d \theta \\
S(h, a, b) & =\frac{b}{2 \pi} \int_{0}^{1} \frac{\Phi\left(h \sqrt{1+a^{2}+a^{2} b^{2} t^{2}}\right) d t}{\left(1+b^{2} t^{2}\right) \sqrt{1+a^{2}+a^{2} b^{2} t^{2}}} \\
\Delta & =1-\rho_{12}^{2}-\rho_{13}^{2}-\rho_{23}^{2}+2 \rho_{12} \rho_{13} \rho_{23}, \\
I_{i j k} & =\rho_{j k}-\rho_{i j} \rho_{i k}, \\
{[k] } & =\left\{\begin{array}{cc}
k & \text { if } k \leq 3 \\
k-3 & \text { if } k>3
\end{array}\right.
\end{aligned}
$$

and $\delta_{h k}=\left\{\begin{array}{ll}0 & \text { if } \operatorname{sgn}(h) \operatorname{sgn}(k)=1 \\ 1 & \text { otherwise }\end{array}\right.$ with $\operatorname{sgn}(h)=\left\{\begin{array}{cc}1 & \text { if } h \geq 0 \\ -1 & \text { if } h<0\end{array}\right.$

For cases $h_{1} \geq 0, h_{2} \geq 0, h_{3}<0$ or $h_{1} \leq 0, h_{2} \leq 0, h_{3}>$ 0 , we have [22]

$$
\begin{aligned}
\Phi\left(h_{1}, h_{2}, h_{3} ; \rho_{12}, \rho_{13}, \rho_{23}\right) \\
=\frac{1}{2}\left[\Phi\left(h_{1}\right)+\Phi\left(h_{2}\right)-\delta_{h_{1} h_{2}}\right]-T\left(h_{1}, a_{1}\right)-T\left(h_{1}, c_{1}\right) \\
\quad-\Phi\left(h_{1}, h_{2},-h_{3} ; \rho_{12},-\rho_{13},-\rho_{23}\right) .
\end{aligned}
$$

Note that for the special case when $h_{1}=h_{2}=h_{3}=h$ and all the correlation factors equal to $\rho$, the trivariate cdf is given by

$$
\begin{aligned}
\Phi(h, h, h ; \rho)= & \frac{3}{2} \Phi(h)-3 T\left(h, \sqrt{\frac{1-\rho}{1+\rho}}\right) \\
& -6 S\left(h, \sqrt{\frac{1-\rho}{1+\rho}}, \frac{1}{\sqrt{1+2 \rho}}\right) .
\end{aligned}
$$

It should be emphasized that the trivariate cdf in this case is a sum of simple finite-range integrals. 


$$
\begin{aligned}
& a_{k}=\frac{h_{[k+1]}-h_{k} \rho_{k[k+1]}}{h_{k} \sqrt{1-\rho_{k[k+1]}^{2}}}, \quad c_{k}=\frac{h_{[k+2]}-h_{k} \rho_{k[k+2]}}{h_{k} \sqrt{1-\rho_{k[k+2]}^{2}}}, \\
& b_{k}=\frac{\left(1-\rho_{k[k+1]}^{2}\right)\left(h_{[k+2]}-h_{k} \rho_{k[k+2]}\right)-I_{k[k+1][k+2]}\left(h_{[k+1]}-h_{k} \rho_{k[k+1]}\right)}{\left(h_{[k+1]}-h_{k} \rho_{k[k+1]}\right) \Delta^{1 / 2}} \text {, } \\
& d_{k}=\frac{\left(1-\rho_{k[k+2]}^{2}\right)\left(h_{[k+1]}-h_{k} \rho_{k[k+1]}\right)-I_{k[k+1][k+2]}\left(h_{[k+2]}-h_{k} \rho_{k[k+2]}\right)}{\left(h_{[k+2]}-h_{k} \rho_{k[k+2]}\right) \Delta^{1 / 2}} .
\end{aligned}
$$

$$
\begin{aligned}
F_{X_{\max }}(x) & =\operatorname{Pr}\left[X_{\max } \leq x\right]=\operatorname{Pr}\left[\bigcap_{k=1}^{n}\left(X_{k} \leq x\right)\right] \\
& =\int_{-\infty}^{x} \cdots \int_{-\infty}^{x}(2 \pi)^{-n / 2}|\mathbf{R}|^{-1 / 2} \exp \left\{-\frac{1}{2}(\mathbf{x}-\boldsymbol{\mu})^{T} \mathbf{R}^{-1}(\mathbf{x}-\boldsymbol{\mu})\right\} d \mathbf{x}
\end{aligned}
$$

\section{Distribution of the maximum of normal random variables}

If $X_{\max }=\max \left\{X_{1}, X_{2}, \cdots, X_{n}\right\}$, the cdf is given by (13). For an arbitrary correlation matrix $\mathbf{R}$, there is no closedform solution for this integral despite longstanding efforts of researchers in the probability and statistics community. Numerically evaluating an $n$-dimensional integral is not satisfactory for large $n$. For special cases $n=2$ and $n=3$, [23] and (7) completely solve this integral. When four or more normal RVs exist, the computational problem becomes difficult and motivates the development of the bounds.

\section{BOUNDS ON CORRELATED LOGNORMAL SUM DISTRIBUTIONS}

We consider the lognormal RV $\gamma_{i}$ to be represented as

$$
\gamma_{i}=10^{Y_{i} / 10}=e^{X_{i}}
$$

where $Y_{i} \sim N\left(m_{Y_{i}}, \sigma_{Y_{i}}^{2}\right), X_{i} \sim N\left(\mu_{i}, \sigma_{i}^{2}\right)$ and $m_{Y_{i}}$ and $\sigma_{Y_{i}}$ are measured in decibels $\left(\mu_{i}=\lambda m_{Y_{i}}\right.$ and $\sigma_{i}=\lambda \sigma_{Y_{i}}$ where $\lambda=0.1 \ln (10)=0.23026)$. In a mobile environment, the $\mathrm{dB}$ spread $\sigma_{Y_{i}}$ can vary between 6 and $12 \mathrm{~dB}$ [2]. For notational convenience, we always use $\gamma_{i}=e^{X_{i}}$.

We primarily intend to derive bounds for lognormal sum distributions and common bounding techniques fail in this case. For example, the Chernoff bounding technique requires the mgf of each summand and this mgf does not exist in closed-form for lognormal sums. This highlights the difficulty of the task facing us. Fortunately, Slimane [24] has suggested that

$$
e^{X_{\max }} \leq I \leq n e^{X_{\max }}
$$

Consequently, the complementary cdf of $I$ is bounded by

$$
1-F_{X_{\max }}(\ln z) \leq P(I>z) \leq 1-F_{X_{\max }}\left[\ln \left(\frac{z}{n}\right)\right]
$$

Our task at hand is to find the cdf of the maximum of the summands.

\section{A. Two lognormal summands}

The cdf of the maximum is readily turned to be

$$
\begin{aligned}
F_{X_{\max }}(x) \\
=\frac{1}{2 \pi \sqrt{1-\rho^{2}}} \\
\quad \cdot \int_{-\infty}^{\frac{x-\mu_{1}}{\sigma_{1}}} \int_{-\infty}^{\frac{x-\mu_{2}}{\sigma_{2}}} \exp \left[-\frac{x_{1}^{2}-2 \rho x_{1} x_{2}+x_{2}^{2}}{2\left(1-\rho^{2}\right)}\right] d x_{1} d x_{2} \\
=\Phi\left(\frac{x-\mu_{1}}{\sigma_{1}}, \frac{x-\mu_{2}}{\sigma_{2}} ; \rho\right) .
\end{aligned}
$$

Since $\Phi\left(h_{1}, h_{2} ; \rho\right)$ can be computed as a sum of single-fold integrals [23], the bounds (16) can be readily computed.

\section{B. Three lognormal summands}

The cdf of the maximum now given by

$$
F_{X_{\max }}(x)=\Phi\left(\frac{x-\mu_{1}}{\sigma_{1}}, \frac{x-\mu_{2}}{\sigma_{2}}, \frac{x-\mu_{3}}{\sigma_{3}} ; \rho_{12}, \rho_{13}, \rho_{23}\right) \text {. }
$$

This provides the distribution of the maximum for the most general use (i.e. non-identically distributed and arbitrarily correlated).

\section{Equally-correlated Lognormal Summands}

We assume that the $X_{i}$ 's in (1) are equally correlated and of the same variance but of different means. The means, variance and covariances are thus given by [29]

(1) $E\left(X_{i}\right)=\mu_{i}, \quad i=1, \cdots, n$,

(2) $E\left[\left(X_{i}-\mu_{i}\right)^{2}\right]=\sigma^{2}, \quad i=1, \cdots, n$,

$$
E\left[\left(X_{i}-\mu_{i}\right)\left(X_{j}-\mu_{j}\right)\right]=\rho \sigma^{2}, \quad i \neq j, i=1, \cdots, n .
$$

The quantity $\rho$ is the correlation coefficient between any two summands in $I$. Note that the equally-correlated fading model is not exactly valid for many scenarios encountered in wireless communications. However, it can be used to described the correlation among equally-spaced (close) antennas and also serves as a worst-case bench mark [30].

A set of equally-correlated normal RVs can be represented as

$$
X_{i}=\sigma \sqrt{\rho} Z_{0}+\sigma \sqrt{1-\rho} Z_{i}+\mu_{i}
$$


for $\rho \geq 0, i=1, \cdots, n$, where $Z_{i}(0 \leq i \leq n)$ are independent standard normal RVs. Note that the equal correlation among the $X_{i}$ 's is ensured by the presence of $Z_{0}$ in all of them. We use the following 'trick' in the subsequent development of various results. The key idea is to fix $Z_{0}$ and the $X_{i}$ 's then become conditionally independent RVs. Distributional results for the conditionally independent variates can be obtained and, as a final step, they have to be averaged over the distribution of $Z_{0}$. This approach, borrowed from Owen and Steck [29], is now extended to compute the cdf of the maximum of correlated normal RVs and joint pdf's of the order statistics of correlated normal RVs (subsection D).

Consequently, let $Z_{0}=t$, then the $X_{i}$ 's are conditionally independent RVs with $X_{i \mid t} \sim N\left[\mu_{i}+\sigma \sqrt{\rho} t, \sigma^{2}(1-\rho)\right]$. Averaging over the distribution of $t$, the cdf of the maximum readily follows as

$$
F_{X_{\max }}(x)=\int_{-\infty}^{\infty}\left[\prod_{i=1}^{n} \Phi\left(\frac{x-\mu_{i}-\sigma \sqrt{\rho} t}{\sigma \sqrt{1-\rho}}\right)\right] \phi(t) d t .
$$

Integral (21) can be evaluated using the Gauss-Hermite quadrature formula [31]. Using (16) and (21), the lower and upper bounds on the complementary cdf are obtained as

$$
\begin{gathered}
1-\int_{-\infty}^{\infty}\left[\prod_{i=1}^{n} \Phi\left(\frac{\ln z-\mu_{i}-\sigma \sqrt{\rho} t}{\sigma \sqrt{1-\rho}}\right)\right] \phi(t) d t \leq P(I>z) \\
\leq 1-\int_{-\infty}^{\infty}\left[\prod_{i=1}^{n} \Phi\left(\frac{\ln (z / n)-\mu_{i}-\sigma \sqrt{\rho} t}{\sigma \sqrt{1-\rho}}\right)\right] \phi(t) d t .
\end{gathered}
$$

Notice that, for independent and identically distributed lognormal variates $(\rho=0)$ with the zero means, (22) reduces to

$$
1-\left[\Phi\left(\frac{\ln z}{\sigma}\right)\right]^{n} \leq P(I>z) \leq 1-\left[\Phi\left(\frac{\ln (z / n)}{\sigma}\right)\right]^{n}
$$

which are identical to the bounds given in [24]. In particular, the lower bound is Farley's approximation [1] [2]. Thus, the lower bound in (22) generalizes Farley's approximation to equally-correlated lognormal summands.

To examine the tightness of the lower and upper bounds, (22) is plotted in Fig. 1 for sums of six lognormal variates $(n=6)$ with $4 \mathrm{~dB}$ and $8 \mathrm{~dB}$ spreads for correlation coefficient values 0.25 and 0.75 . The simulation results are also provided for comparison. The lower bound is tighter than the upper bound, similar to the bounds for independent lognormal variates [24], and it becomes tighter as the standard deviation increases. As expected, the lower bound loses its accuracy when $\rho$ increases because the sum distribution is no longer dominated by the maximum summand due to the high correlation among the summands. Finally, the lower bound is accurate provided $\rho$ is small. This behavior is reminiscent of the Farley bound for the independent case.

\section{Improved Bounds for identically distributed, equally- correlated lognormal summands}

The bounds derived in the previous section can be improved, albeit at a cost of computational complexity. Improved bounds

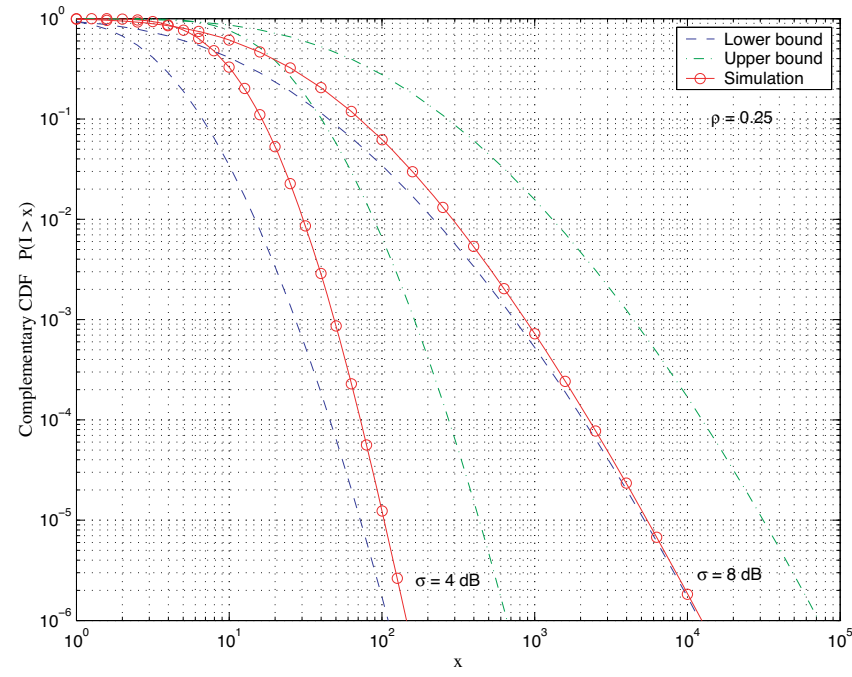

Fig. 1. Lower and upper bounds for the distribution of a sum of six equallycorrelated lognormal RVs. The normalized correlation is $\rho=0.25$.

can be obtained by considering the order statistics of one more summand as [24]

$$
I \geq e^{X_{\max }}+(n-1) e^{X_{\min }} .
$$

In order to find the distribution of the improved lower bound, the joint pdf of $X_{\min }$ and $X_{\max }$ is required. We use the representation (20) with $\mu_{i}=\mu$ and $Z_{0}=t$. When $Z_{0}$ is fixed $X_{\min }$ and $X_{\max }$ are determined by $Z_{\min }$ and $Z_{\max }$. Since $Z_{i}(i=0, \cdots, n)$ are independent $N(0,1)$, the conditional joint pdf of $X_{\min }$ and $X_{\max }$ can be written as [32, Eq.2.21]

$$
\begin{aligned}
& f_{X_{\min } X_{\max }}\left(y, z \mid Z_{0}=t\right) \\
& \quad=\frac{1}{\sigma^{2}(1-\rho)} \frac{n !}{(n-2) !}[\Phi(\tilde{z})-\Phi(\tilde{y})]^{n-2} \phi(\tilde{y}) \phi(\tilde{z}), \quad y \leq z
\end{aligned}
$$

where $\tilde{\sharp}=\frac{\sharp-\sigma \sqrt{\rho} t-\mu}{\sigma \sqrt{1-\rho}}$.

In a similar manner, the improved upper bound on a correlated lognormal sum is given by applying the following order statistics [24]

$$
I \leq e^{X_{\max }}+(n-1) e^{X_{(n-1)}}
$$

where $X_{(n-1)}$ is the second largest of $X_{1}, X_{2}, \cdots, X_{n}$. Again by fixing $Z_{0}$ in (20), the conditional joint pdf of $X_{(n-1)}$ and $X_{\max }$ is given by

$$
\begin{aligned}
& f_{X_{(n-1)} X_{\max }}\left(y, z \mid Z_{0}=t\right) \\
& \quad=\frac{n !}{\sigma^{2}(1-\rho)(n-2) !} \Phi(\tilde{y})^{n-2} \phi(\tilde{y}) \phi(\tilde{z}), \quad y \leq z .
\end{aligned}
$$

Using (24), (25), (26) and (27), tighter lower and upper bounds on the complementary cdf of a sum of correlated lognormal RVs can be obtained by

$$
\begin{aligned}
& P(I>x) \\
& \geq 1-\int_{-\infty}^{\infty} \int_{-\infty}^{\ln x} \int_{-\infty}^{y_{m}} f_{X_{\min } X_{\max }}\left(y, z \mid Z_{0}=t\right) \phi(t) d y d z d t
\end{aligned}
$$




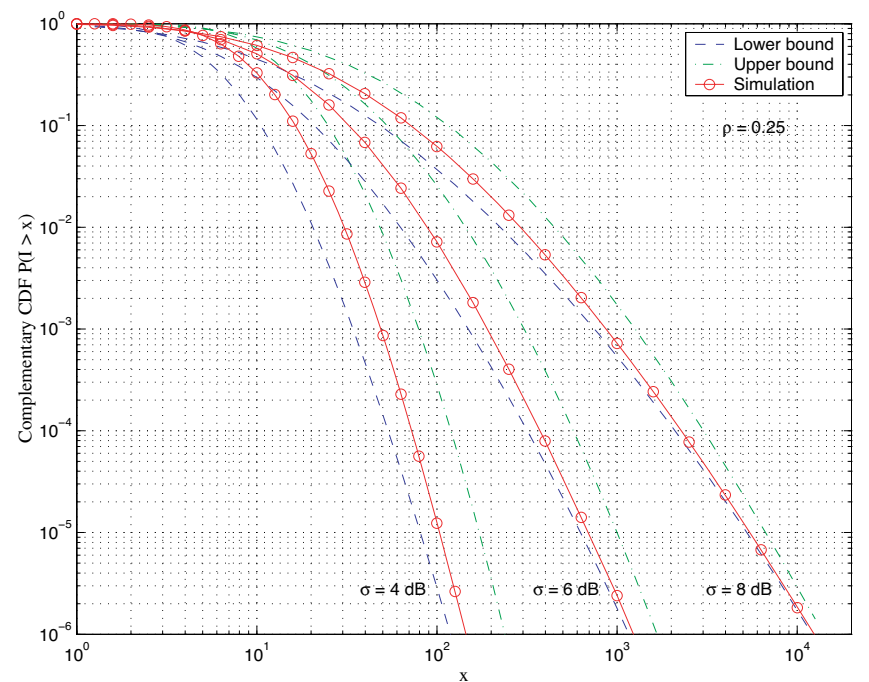

Fig. 2. Improved lower and upper bounds for the distribution of a sum of six equally-correlated lognormal RVs. The normalized correlation is $\rho=0.25$.

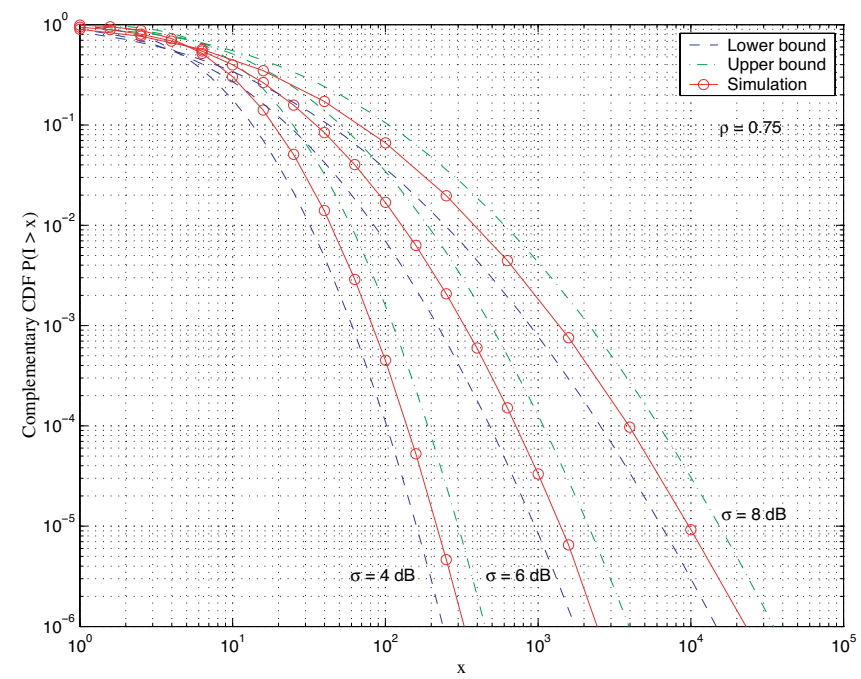

Fig. 3. Improved lower and upper bounds for the distribution of a sum of six equally-correlated lognormal RVs. The normalized correlation is $\rho=0.75$.

and

$$
\begin{aligned}
& P(I>x) \\
& \leq 1-\int_{-\infty}^{\infty} \int_{-\infty}^{\ln x} \int_{-\infty}^{y_{m}} f_{X_{(n-1)} X_{\max }}\left(y, z \mid Z_{0}=t\right) \phi(t) d y d z d t
\end{aligned}
$$

where

$$
y_{m}= \begin{cases}z & z<\ln (x / n) \\ \ln \left[\left(x-e^{z}\right) /(n-1)\right] & z \geq \ln (x / n) .\end{cases}
$$

It is observed that when $\rho=0$, both (25) and (27) reduce to the joint pdf of ordered variates for independent summands and (28a) and (28b) reduces to two bounds in [24]. Note that the bounds which are three-fold integrals pose numerical difficulties. Appendix I shows that (28a) and (28b) can be simplified as (29).

To examine the tightness of these new lower and upper bounds, (29) is computed for six summands and dB spreads of 4 and 8 (same as in the previous section). These bounds for correlation coefficient values 0.25 and 0.75 are shown in Figs. 2 and 3 along with the simulation results. The two new bounds are tighter than (22). Although the improved bounds are more complex, their computation time does not increase substantially compared to (22).

\section{Applications}

The previous expressions for the cdf of $X_{\max }$ can be used to compute the performance of selection combiner (SC) receivers in correlated lognormal fading. Let the branch SNR's be given by $\gamma_{i}=e^{X_{i}}(i=1,2, \cdots, n)$. The maximum of the branch $\mathrm{SNR}$ is the output of $\mathrm{SC}$ receiver as

$$
\gamma_{\mathrm{sc}}=\max \left\{\gamma_{1}, \gamma_{2}, \cdots, \gamma_{n}\right\}
$$

We are interested in the moments of the SC outputs given by

$$
E\left[\gamma_{\mathrm{sc}}^{k}\right]=\int_{0}^{\infty} x^{k} f_{\gamma_{\mathrm{sc}}}(x) d x \quad k=1,2, \cdots .
$$

Although SC performance has been treated for a wide variety of channel models, performance over correlated lognormal fading channels is not available, except for the dual-diversity case by Simon and Alouini [26]. For the equally-correlated case, expressing the moments as a two-fold integral using (21), we find that $E\left[\gamma_{\mathrm{sc}}^{k}\right]$ can be represented as (32). In general, there is no closed-form solution for this double integral. However, when all the means are identical, using the substitution $y=\frac{x-\mu-\sigma \sqrt{\rho} t}{\sigma \sqrt{1-\rho}}$, the $k$-th moment is found to be

$$
\begin{aligned}
& E\left[\gamma_{\mathrm{sc}}^{k}\right] \\
& =n \int_{-\infty}^{\infty} \int_{-\infty}^{\infty} e^{k(\sigma \sqrt{1-\rho} y+\mu+\sigma \sqrt{\rho} t)}[\Phi(y)]^{n-1} \phi(y) \phi(t) d y d t \\
& =n e^{k \mu+k^{2} \sigma^{2} / 2} \int_{-\infty}^{\infty}[\Phi(y+k \sigma \sqrt{1-\rho})]^{n-1} \phi(y) d y .
\end{aligned}
$$

Thus, all SC output moments are derived as single-fold integrals of computable functions. For the dual-branch case ( $n=2$ ), (33) can be interpreted as a probability integral involving two independent $N(0,1)$ variables, say, $X_{1}$ and $X_{2}$ :

$$
\begin{aligned}
E\left[\gamma_{\mathrm{sc}}^{k}\right] & =2 e^{k \mu+k^{2} \sigma^{2} / 2} \int_{-\infty}^{\infty} \Phi(y+k \sigma \sqrt{1-\rho}) \phi(y) d y \\
& =2 e^{k \mu+k^{2} \sigma^{2} / 2} \operatorname{Pr}\left\{X_{1}<X_{2}+k \sigma \sqrt{1-\rho}\right\} \\
& =2 e^{k \mu+k^{2} \sigma^{2} / 2} \Phi\left(k \sigma \sqrt{\frac{1-\rho}{2}}\right) .
\end{aligned}
$$

This is exactly the correlated identically distributed fading result given in [26, Eq. 34]. Closed-form expressions for $n=3$ and $n=4$ cases can also be derived. Using [33] and (33), for equally-correlated identically distributed 3-branch systems, the $\mathrm{SC}$ output moments are given by

$$
\begin{aligned}
E\left[\gamma_{\mathrm{sc}}^{k}\right]= & 3 e^{k \mu+k^{2} \sigma^{2} / 2} \\
& \cdot\left[\Phi\left(k \sigma \sqrt{\frac{1-\rho}{2}}\right)-2 T\left(k \sigma \sqrt{\frac{1-\rho}{2}}, \frac{1}{\sqrt{3}}\right)\right] .
\end{aligned}
$$




$$
\begin{array}{r}
1-\int_{-\infty}^{\infty} \int_{-\infty}^{\ln x} \frac{n !}{(n-2) ! \sigma \sqrt{1-\rho}}\left[\sum_{r=0}^{n-2}\left(\begin{array}{c}
n-2 \\
r
\end{array}\right) \frac{(-1)^{r}}{r+1} \Phi(\tilde{z})^{n-2-r} \phi(\tilde{z}) \Phi\left(y^{*}\right)^{r+1}\right] \phi(t) d z d t \\
\leq P(I>x) \leq 1-\int_{-\infty}^{\infty} \int_{-\infty}^{\ln x} \frac{n}{\sigma \sqrt{1-\rho}} \Phi\left(y^{*}\right)^{n-1} \phi(\tilde{z}) \phi(t) d z d t .
\end{array}
$$

$$
E\left[\gamma_{\mathrm{sc}}^{k}\right]=\frac{1}{\sigma \sqrt{1-\rho}} \sum_{i=1}^{n} \int_{-\infty}^{\infty} \int_{-\infty}^{\infty} e^{x k} \prod_{\substack{j=1 \\ j \neq i}}^{n} \Phi\left(\frac{x-\mu_{j}-\sigma \sqrt{\rho} t}{\sigma \sqrt{1-\rho}}\right) \phi\left(\frac{x-\mu_{i}-\sigma \sqrt{\rho} t}{\sigma \sqrt{1-\rho}}\right) \phi(t) d t d x
$$

$$
E\left[\gamma_{\mathrm{sc}}^{k}\right]=4 e^{k \mu+k^{2} \sigma^{2} / 2}\left[\frac{3}{2} \Phi\left(k \sigma \sqrt{\frac{1-\rho}{2}}\right)-3 T\left(k \sigma \sqrt{\frac{1-\rho}{2}}, \frac{1}{\sqrt{3}}\right)-6 S\left(k \sigma \sqrt{\frac{1-\rho}{2}}, \frac{1}{\sqrt{3}}, \frac{1}{\sqrt{2}}\right)\right] .
$$

Using [33] and (33), for equally-correlated identically distributed 4-branch case, the SC output moments are given by (36).

To the best of our knowledge, (33) and (34) are new results and they enable rapid performance evaluation of 3-branch and 4-branch SC over correlated lognormal channels.

Amount of fading (AF), which is the variance normalized by the square of the mean, is sometimes used as a performance measure for diversity systems [26]. For a diversity system with output SNR $\gamma_{\text {out }}$, the AF measure is given by

$$
\mathrm{AF}_{\text {out }}=\frac{E\left[\gamma_{\text {out }}^{2}\right]-\left[E\left(\gamma_{\text {out }}\right)\right]^{2}}{\left[E\left(\gamma_{\text {out }}\right)\right]^{2}}
$$

The AF measure for three-branch SC over equally-correlated lognormal fading is given by

$\mathrm{AF}_{\mathrm{sc}}=\frac{e^{\sigma^{2}}}{3} \frac{\left[\Phi(\sigma \sqrt{2(1-\rho)})-2 T\left(\sigma \sqrt{2(1-\rho)}, \frac{1}{\sqrt{3}}\right)\right]}{\left[\Phi\left(\sigma \sqrt{\frac{1-\rho}{2}}\right)-2 T\left(\sigma \sqrt{\frac{1-\rho}{2}}, \frac{1}{\sqrt{3}}\right)\right]^{2}}-1$

for $n=3$. For four-branch SC over equally-correlated lognormal fading, the AF is given by (39). To the best of our knowledge, (36) and (37) are new AF expressions for SC over correlated lognormal channels.

For comparison, the MRC output moments are derived next. The MRC output is given by $\gamma_{\mathrm{mrc}}=\sum_{i=1}^{n} e^{X_{i}}$. Expanding $\gamma_{\mathrm{mrc}}^{k}$ multinomially, the $k$-th moment is found to be

$$
E\left[\gamma_{\mathrm{mrc}}^{k}\right]=\sum_{k_{1}+\cdots+k_{n}=k}\left(\begin{array}{c}
k \\
k_{1} \cdots k_{n}
\end{array}\right) E\left[e^{k_{1} X_{1}+\cdots+k_{n} X_{n}}\right]
$$

where the summation is to be carried over all non-negative integers $k_{1}, k_{2}, \cdots, k_{n}$ that sum to $k$ and $\left(\begin{array}{c}n \\ k_{1} \cdots k_{n}\end{array}\right)=\frac{n !}{k_{1} ! \cdots k_{n} !}$. The expectation in (40) can be readily recognized as the mgf of the multivariate normal distribution (3). We thus find that

$$
E\left[\gamma_{\mathrm{mrc}}^{k}\right]=\sum_{k_{1}+\cdots+k_{n}=k}\left(\begin{array}{c}
k \\
k_{1} \cdots k_{n}
\end{array}\right) \exp \left\{\mathbf{k}^{T} \boldsymbol{\mu}+\frac{1}{2} \mathbf{k}^{T} \mathbf{R} \mathbf{k}\right\}
$$

where $\mathbf{k}=\left(k_{1}, k_{2}, \cdots, k_{n}\right)$. This expression holds for arbitrary correlation structures and not necessarily identically distributed lognormal RVs and (41) generalizes the MRC moments derived for the dual-branch case in [26, Eq. (13)].

Using $\mathbf{R}=\left[r_{i j}\right]$ where $r_{i j}=\rho_{i j} \sigma_{i} \sigma_{j}$ and (41), we can show that the AF measure for MRC over correlated

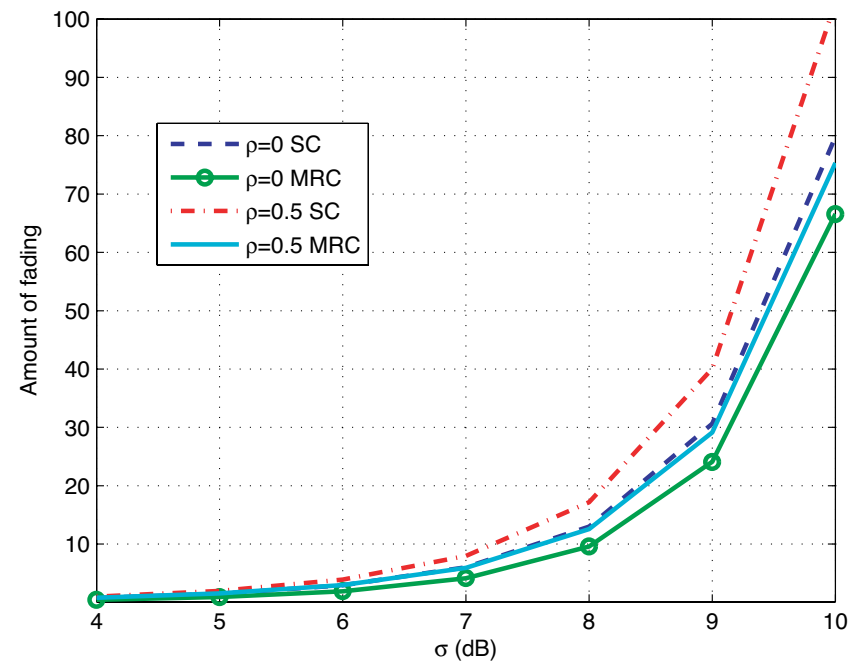

Fig. 4. Amount of fading for 3-branch SC for different correlation coefficient $\rho$.

lognormal channels is given by Eq. (42). Eq. (42) holds for arbitrary correlation structures and not necessarily identically distributed lognormal RVs and it also generalizes the AF measure for MRC derived for the dual-branch case in [26, Eq. (16)]. Fig. 4 shows the AF measure for SC and MRC over correlated lognormal channels as a function of the $\mathrm{dB}$ spread and for $\rho=0,0.5$.

For EGC, the output SNR may be given as

$\gamma_{\text {egc }}=\frac{1}{n}\left(\sqrt{\gamma_{1}}+\cdots+\sqrt{\gamma_{n}}\right)^{2}=\frac{1}{n}\left(e^{\frac{1}{2} X_{1}}+\ldots+e^{\frac{1}{2} X_{n}}\right)^{2}$.

Expanding $\gamma_{\text {egc }}^{k}$ multinomially, it can be shown that

$$
\begin{aligned}
E\left[\gamma_{\mathrm{egc}}^{k}\right] & \\
& =\frac{1}{n} \sum_{k_{1}+\cdots+k_{n}=2 k}\left(\begin{array}{c}
2 k \\
k_{1} \cdots k_{n}
\end{array}\right) E\left[e^{\frac{1}{2} k_{1} X_{1}+\cdots+\frac{1}{2} k_{n} X_{n}}\right] \\
& =\frac{1}{n} \sum_{k_{1}+\cdots+k_{n}=2 k}\left(\begin{array}{c}
2 k \\
k_{1} \cdots k_{n}
\end{array}\right) \exp \left\{\frac{1}{2} \mathbf{k}^{T} \boldsymbol{\mu}+\frac{1}{8} \mathbf{k}^{T} \mathbf{R k}\right\}
\end{aligned}
$$

where the summation is to be carried over all non-negative integers $k_{1}, k_{2}, \cdots, k_{n}$ that sum to $2 k$. Using (42), we can readily derive the AF measure for EGC and this is omitted for brevity. 


$$
\mathrm{AF}_{\mathrm{sc}}=\frac{e^{\sigma^{2}}}{4} \frac{\left[\frac{3}{2} \Phi(\sigma \sqrt{2(1-\rho)})-3 T\left(\sigma \sqrt{2(1-\rho)}, \frac{1}{\sqrt{3}}\right)-6 S\left(\sigma \sqrt{2(1-\rho)}, \frac{1}{\sqrt{3}}, \frac{1}{\sqrt{2}}\right)\right]}{\left[\frac{3}{2} \Phi\left(\sigma \sqrt{\frac{1-\rho}{2}}\right)-3 T\left(\sigma \sqrt{\frac{1-\rho}{2}}, \frac{1}{\sqrt{3}}\right)-6 S\left(\sigma \sqrt{\frac{1-\rho}{2}}, \frac{1}{\sqrt{3}}, \frac{1}{\sqrt{2}}\right)\right]^{2}}-1
$$

$$
\mathrm{AF}_{\mathrm{mrc}}=\frac{\sum_{i} \exp \left[2\left(\mu_{i}+\sigma_{i}^{2}\right)\right]+2 \sum_{i, j} \exp \left(\mu_{i}+\mu_{j}+\frac{1}{2}\left(\sigma_{i}^{2}+\sigma_{j}^{2}\right)+\rho_{i j} \sigma_{i} \sigma_{j}\right)}{\left[\sum_{i} \exp \left(\mu_{i}+\frac{1}{2} \sigma_{i}^{2}\right)\right]^{2}}-1
$$

\section{A. Outage Probabilities}

A widely-used performance measure is the outage, the probability that the diversity combiner output falls below a predefined threshold. The outage of $L$-branch SC $P_{\text {out }}^{\mathrm{sc}}$ is therefore given by

$$
\begin{aligned}
P_{\mathrm{out}}^{\mathrm{sc}}\left(\gamma_{\mathrm{th}}\right) & =P\left[\gamma_{\mathrm{sc}}<\gamma_{\mathrm{th}}\right] \\
& =\int_{-\infty}^{\infty} \prod_{i=1}^{L} \Phi\left(\frac{\ln \gamma_{\mathrm{th}}-\mu_{i}-\sigma \sqrt{\rho} t}{\sigma \sqrt{1-\rho}}\right) \phi(t) d t .
\end{aligned}
$$

Again there is no closed-form solution in general. For $L=2$ and 3 and for identical means, however we get Eq. (46) for the outage probability using [33]:

\section{CONCLUSION}

While lognormal sums widely occur in wireless applications, the sum distribution problem remains unsolved. Even for independent sums, let alone for the correlated case, a closed-form solution has been elusive. This letter therefore has derived upper and lower bounds for the distribution of a sum of 2 or 3 lognormal RVs with arbitrary correlation and of a sum of any number of equally-correlated lognormal RVs. The bounds are single-fold integrals of readily computable functions. Several previously known bounds for the independent case are shown to be special cases of these results. An improved set of bounds are also derived which are expressed as 2-fold integrals. The distributional results developed for bounding purposes are further utilized to obtain several performance measures for MRC, SC and EGC. Thus, the moments of output SNR, outage probability and amount of fading were derived for diversity systems over correlated lognormal environments. A possible future topic is to extend these results to generalized selection combiners. A challenging open problem is to extend our results to arbitrary correlation patterns.

\section{APPENDIX A}

Consider the evaluation of the integration in (28b), the part

$$
\begin{aligned}
I & =\int_{-\infty}^{y_{m}} f_{X_{(n-1)} X_{\max }}\left(y, z \mid Z_{0}=t\right) d y \\
& =\int_{-\infty}^{y_{m}}[1-Q(\tilde{y})]^{n-2} \phi(\tilde{y}) \frac{d \tilde{y}}{d y} d y \\
& =\int_{-\infty}^{y^{*}}[1-Q(\tilde{y})]^{n-2} \phi(\tilde{y}) d \tilde{y} \\
& =\frac{1}{n-1}\left[1-Q\left(y^{*}\right)\right]^{n-1}
\end{aligned}
$$

where $y^{*}=\frac{y_{m}-\sigma \sqrt{\rho} t}{\sigma \sqrt{1-\rho}}$. Thus, $(28 \mathrm{~b})$ is reduced from 3-D to 2-D integration as

$$
\begin{aligned}
& P(I>x) \\
& \quad \leq 1-\int_{-\infty}^{\infty} \int_{-\infty}^{\ln x} \frac{n}{\sigma \sqrt{1-\rho}}\left[1-Q\left(y^{*}\right)\right]^{n-1} \phi(\tilde{z}) \phi(t) d z d t
\end{aligned}
$$

Similar development occurs with (28a) since

$$
[Q(\tilde{y})-Q(\tilde{z})]^{n-2}=\sum_{r=0}^{n-2}\left(\begin{array}{c}
n-2 \\
r
\end{array}\right)(-1)^{r}[1-Q(\tilde{z})]^{n-2-r}[1-Q(\tilde{y})]^{r} .
$$

Then (28a) is reduced to Eq. (A.4).

\section{REFERENCES}

[1] Y. Yeh and S. C. Schwartz, "Outage probability in mobile telephony due to multiple lognormal interferers," IEEE Trans. Commun., vol. 32, no. 4, pp. 380-388, Apr. 1984.

[2] N. C. Beaulieu, A. A. Abu-Dayya, and P. J. McLane, "Estimating the distribution of a sum of independent lognormal random variables," IEEE Trans. Commun., vol. 43, pp. 2869-2873, Dec. 1995.

[3] J. Aitchison and J. A. C. Brown, The Lognormal Distribution. Cambridge University Press, 1957.

[4] L. F. Fenton, "The sum of lognormal probability distributions in scatter transmission systems," IRE Trans. Commun. Syst., vol. CS-8, pp. 57-67, Mar. 1960.

[5] D. C. Schleher, "Generalized Gram-Charlier series with application to the sum of lognormal variates," IEEE Trans. Inform. Theory, pp. 275280, Mar. 1977.

[6] S. C. Schwartz and Y. S. Yeh, "On the distribution function and moments of power sums with lognormal components," Bell Syst. Tech. J., vol. 61, no. 7, pp. 1441-1462, Sept. 1982

[7] P. Cardieri and T. S. Rappaport, "Statistics of the sum of lognormal variables in wireless communications," in Peoc. IEEE Vehicular Technology Conference VTC 2000-Spring, vol. 3, Tokyo, pp. 1823-1827.

[8] N. C. Beaulieu and Q. Xie, "An optimal lognormal approximation to lognormal sum distributions," IEEE Trans. Veh. Technol., vol. 53, no. 2, pp. 479-489, Mar. 2004.

[9] N. C. Beaulieu and F. Rajwani, "Highly accurate simple closed-form approximations to lognormal sum distributions and densities," IEEE Commun. Lett., vol. 8, no. 12, pp. 709-711, Dec. 2004.

[10] C. L. J. Lam and T. Le-Ngoc, "Estimation of typical sum of lognormal random variables using log shifted gamma approximation," IEEE Commun. Lett., vol. 10, no. 4, pp. 234-235, Apr. 2006.

[11] M. Pratesi, F. Santiccu, and F. Graziosi, "Generalized moment matching for the linear combination of lognormal RVs: application to outage analysis in wireless systems," IEEE Trans. Wireless Commun., vol. 5, no. 5, pp. 1122-1132, May 2006.

[12] Q. T. Zhang and S. H. Song, "Model selection and estimation for lognormal sums in Pearson's framework," in Proc. 63rd IEEE Vehicular Technology Conference, 2006 VTC-Spring, vol. 6, Melbourne, Vic., May 2006, pp. 2823-2827. 


$$
P_{\text {out }}^{\mathrm{sc}}\left(\gamma_{\mathrm{th}}\right)= \begin{cases}\Phi\left(\frac{\ln \gamma_{\mathrm{th}}-\mu}{\sigma}\right)-2 T\left(\frac{\ln \gamma_{\mathrm{th}}-\mu}{\sigma}, \sqrt{\frac{1-\rho}{1+\rho}}\right) & L=2 \\ \frac{3}{2} \Phi\left(\frac{\ln \gamma_{\mathrm{th}}-\mu}{\sigma}\right)-3 T\left(\frac{\ln \gamma_{\mathrm{th}}-\mu}{\sigma}, \sqrt{\frac{1-\rho}{1+\rho}}\right)-6 S\left(\frac{\ln \gamma_{\mathrm{th}}-\mu}{\sigma}, \sqrt{\frac{1-\rho}{1+\rho}}, \sqrt{\frac{1}{1+2 \rho}}\right) & L=3 .\end{cases}
$$

$$
P(I>x) \geq 1-\int_{-\infty}^{\infty} \int_{-\infty}^{\ln x} \frac{n !}{(n-2) ! \sigma \sqrt{1-\rho}}\left[\sum_{r=0}^{n-2}\left(\begin{array}{c}
n-2 \\
r
\end{array}\right) \frac{(-1)^{r}}{r+1}[1-Q(\tilde{z})]^{n-2-r} \phi(\tilde{z})\left[1-Q\left(y^{*}\right)\right]^{r+1}\right] \phi(t) d z d t
$$

[15] N. B. Mehta, J. Wu, A. F. Molisch, and J. Zhang, "Approximating a Sum of Random Variables with a Lognormal," IEEE Trans. Wireless Commun., vol. 6, no. 7, pp. 2690-2699, July 2007.

[16] L. Zhao and J. Ding, "Least Squares Approximations to Lognormal Sum Distributions," IEEE Trans. Veh. Technol., vol. 56, no. 2, pp. 991-997, Mar. 2007.

[17] C. Fischione, F. Graziosi, and F. Santucci, "Approximation for a sum of on-off lognormal processes with wireless applications," IEEE Trans. Commun., vol. 55, no. 10, pp. 1984-1993, Oct. 2007.

[18] J. Zhang and V. Aalo, "Effect of macrodiversity on average-error probabilities in a Rician fading channel with correlated lognormal shadowing," IEEE Trans. Commun., vol. 49, no. 1, pp. 14-18, Jan. 2001.

[19] A. A. Abu-Dayya and N. C. Beaulieu, "Outage probabilities in the presence of correlated lognormal interferers," IEEE Trans. Veh. Technol., vol. 43, pp. 164-173, Feb. 1994.

[20] M. Pratesi, F. Santucci, F. Graziosi, and M. Ruggieri, "Outage analysis in mobile radio systems with generically correlated lognormal interferers," IEEE Trans. Commun., vol. 48, no. 3, pp. 381-385, Mar. 2000.

[21] A. Ligeti, "Outage probability in the presence of correlated lognormal useful and interfering components," IEEE Commun. Lett., vol. 4, no. 1, pp. 15-17, Jan. 2000.

[22] G. P. Steck, "A table for computing trivariate normal probabilities," Annals of Mathematical Statistics, vol. 29, pp. 780-800, 1958.

[23] D. B. Owen, "Tables for computing bivariate normal probabilities," Annals of Mathematical Statistics, vol. 27, pp. 1075-1090, 1956.

[24] S. B. Slimane, "Bounds on the distribution of a sum of independent lognormal random variables," IEEE Trans. Commun., vol. 49, pp. 975978, June 2001

[25] F. Berggren and S. Slimane, "A simple bound on the outage probability with lognormally distributed interferers," IEEE Commun. Lett. (USA), vol. 8, no. 5, pp. 271-3, May 2004.

[26] M. S. Alouini and M. K. Simon, "Dual diversity over correlated lognormal fading channels," IEEE Trans. Commun., vol. 50, pp. 1946-1959, Dec. 2002.

[27] S. Kotz, N. Balakrishnan, and N. L. Johnson, Continuous Multivariate Distributions, 2nd ed. New York: John Wiley \& Sons, 2000, vol. v.1.

[28] M. K. Simon, "A simpler form of the Craig representation for the twodimensional joint Gaussian Q-function," IEEE Commun. Lett., vol. 6 , pp. 49-51, Feb. 2002.

[29] D. B. Owen and G. P. Steck, "Moments of order statistics from the equicorrelated multivariate normal distribution," Ann. Math. Statist., vol. 33, pp. 1286-1291, 1962.

[30] V. A. Aalo, "Performance of maximal-ratio diversity systems in a correlated Nakagami-fading environment," IEEE Trans. Commun., vol. 43, no. 8, pp. 2360-2369, Aug. 1995.

[31] M. Abramowitz and I. A. Stegun, Handbook of Mathematical Functions. New York: Dover Publications, 1972.

[32] H. A. David, Order Statistics, 2nd ed. New York: John Wiley \& Sons, Inc., 1981.

[33] D. B. Owen, "A table of normal integrals," Comm. Statist. B-Simulation Comput., vol. 9, no. 4, pp. 389-419, 1980. 